\title{
An Overview of Quality of Life in Vitiligo Patients - At Glance
}

\author{
Ayesha Mateen ${ }^{1}$, Rabbani Syed ${ }^{1,2 *}$, Khalid Khalaf Alharbi ${ }^{2}$ and Imran Ali Khan ${ }^{2}$
}

${ }^{1}$ Department of Biotechnology, Central Research Institute of Unani Medicine, Hyderabad, India

${ }^{2}$ Department of Clinical Laboratory Sciences, College of Applied Medical Sciences, King Saud University, Riyadh, Kingdom of Saudi Arabia

\begin{abstract}
Vitiligo is an acquired depigmentary skin disorder characterized by white maculae on the skin associated with social stigma. Vitiligo has major impact on the quality of life due its high cosmetic value, chronic nature of disease and long term ineffective treatments. Vitiligo patients suffer from low self-esteem and poor body image that affects their emotional and psychological well-being. To investigate quality of life of Vitiligo patients, psychological impact of unpredictable course of disease and long term treatment among South Indian population. A total of 155 Vitiligo patients, undertaking treatment in dermatology clinic at the Central Research Institute of Unani Medicine (CRIUM), who completed the Dermatology Quality of Life Index (DLQI) questionnaire, participated in this study. Patients with Vitiligo averaged at about $27.4 \%$ of maximum psychological impairment as measured by the DLQI summary score. Disability in patients was highest in treatment category with $54.6 \%$ of impairment followed by symptoms and feelings $(41.83 \%)$. Dark skin, unmarried, slow responders to the treatment and those having chronicity more than 5 years showed worse quality of life and had $70 \%, 61 \%, 62 \%$ and $76.5 \%$ of impairment in the treatment front of DLQI respectively when compared to light skin $(50 \%)$, married $(52.5 \%)$, rapid responders $(26 \%)$ and chronicity less than 5 years $(52 \%)$. There was no significant difference in the quality of life between male and female Vitiligo patients $(p=0.88)$. Our results show that Vitiligo patients suffer in all aspects of life especially in treatment aspect of DLQI. Psychological impairment strongly affects the social well-being of Vitiligo patients.
\end{abstract}

Keywords: Vitiligo; Quality of life; DLQI

\section{Introduction}

Vitiligo, also known as leukoderma, is an acquired depigmentary disorder characterized by the appearance of white patches resulting from the loss of functional melanocytes and melanin from the skin [1]. Vitiligo is an acquired, idiopathic, and worldwide common depigmentation disorder with an estimated prevalence from 0.1 to $8 \%$. These numbers are based on clinical population studies and field research examining inhabitants of geographically enclosed areas. The often cited prevalence of $8 \%$ could not be confirmed after excluding clinical patient populations. Accordingly, the worldwide prevalence of Vitiligo ranges between 0.5 and 2\% [2]. Adults and children of both sexes are equally affected; the proportion of patients with a positive family history varies from one part of the world to another, with particularly wide ranges reported in India (25-18\%), with reports of up to $40 \%$ elsewhere in the world [3]. Some dermatological outpatient records show the incidence of vitiligo to be $3 \%$ to $4 \%$ in India although an incidence as high as $8.8 \%$ has also been reported [4]. The exact aetiology of Vitiligo is unknown, but four main theories exist to explain it: the autoimmune hypothesis, the neural hypothesis, the selfdestruct hypothesis, and the growth factor defect hypothesis, but none satisfactory. It is believed that Vitiligo is a polygenic trait and that a convergence theory, combining elements of different theories across a spectrum of expression is the most accurate aetiology [5]. Vitiligo is not a physically damaging disease; other than an increased sensitivity to UV radiation most of the disease effects are social and psychological, especially for dark-skinned races. There are both surgical and nonsurgical treatments for Vitiligo but fails to give cosmetically acceptable pigmentation [6].

Vitiligo is an acquired depigmenting disorder characterized by the loss of functional melanocytes from the epidermis [7], Vitiligo is in most cases diagnosed clinically, and so obvious are the features of a well-developed lesion of this disease. Other investigations are rarely required. However, occasionally Vitiligo especially in its early evolving stage or in some of its localised expressions may pose diagnostic difficulties as the lesions are hypo pigmented rather than depigmented and other differential diagnoses may be considered [8]. Vitiligo is of great cosmetic concern since the change in appearance caused by
Vitiligo can affect a person's emotional and psychological well-being. Since ancient times patients with Vitiligo suffered the same mental abuses as lepers. In India young woman with Vitiligo have little chance of getting married. Appearance of skin can condition an individual self-image, and any pathological alteration can have psychological consequences. Implications of Vitiligo ruin young girls who are almost condemned of spinsterhood and at many occasions these patients are denied of first line jobs [9]. Vitiligo is thus an important skin disease having major impact on the quality of life; therefore we intended to study the psychological impairment in different aspect of life in Vitiligo patients. This analysis describes self-reported dermatological life quality index (DLQI) of patients with segmental and non-segmental Vitiligo.

\section{Methodology}

A total of 155 patients undergoing treatment for Vitiligo in Dermatology clinic at the Central Research Institute of Unani Medicine (CRIUM) consented for the study and completed the Dermatology Life Quality Index (DLQI) questionnaire (with permission from Wales College of medicine, Dermatology department). Study subjects were over 16 years of age, of either sex, with Segmental Vitiligo (SV) or Non-Segmental Vitiligo (NSV) types. The study was approved by the CRIUM ethical committee. DLQI questionnaire measures six diseasespecific domains, i.e., symptoms and feelings, leisure activities, daily activities, work and school, personal relationships, and treatment. Individual items are summed to generate an overall DLQI score [10]. Scores are expressed as percentages, with higher percentage indicating

*Corresponding author: Rabbani Syed, Department of Clinical Laboratory Sciences, College of Applied Medical Sciences, King Saud University, Riyadh, Kingdom of Saudi Arabia, E-mail: rabbanisyd@gmail.com

Received April 20, 2015; Accepted June 16, 2015; Published June 28, 2015

Citation: Mateen A, Syed R, Alharbi KK, Khan IA (2015) An Overview of Quality of Life in Vitiligo Patients - At Glance. Pigmentary Disorders S3: 003. doi:10.4172/2376-0427.S3-003

Copyright: $\odot 2015$ Mateen A, et al. This is an open-access article distributed under the terms of the Creative Commons Attribution License, which permits unrestricted use, distribution, and reproduction in any medium, provided the original author and source are credited. 
greater impairment in the quality of life. Results were reported for the total group and for subgroups.

\section{Statistical Analysis}

Data were stored and analysed using SPSS 19 (Statistical Package for Social Science; release 19.0). Chi-square test and Fishers exact test were used and a $\mathrm{P}$-value $<0.05$ was considered as significant

\section{Results}

The mean age of patients is $32.4 \pm 13.9$ and having a mean chronicity of $3.09 \pm 5.01$ yrs. The demographic characteristics of the total analysis group and the subgroups are described and DLQI scores are displayed. Patients with Vitiligo averaged at about $27.4 \%$ of maximum impairment as measured by the DLQI summary score. Vitiligo patients scored highest in the treatment component of the DLQI, which measures pain, stress and difficulties faced in getting the right kind of treatment, with $56.1 \%$ of impairment reported. This was followed by symptoms and feelings, with $41.83 \%$ of impairment measuring the embarrassment and suffering associated with the disease. Impairment in work and school, and leisure activities ranged from $9.9 \%$ to $17 \%$. Males and females were equally affected $(8.25 \pm 5.05$ vs $8.13 \pm 4.69$, $\mathrm{p}=0.8$ ). Patients with non-segmental type consistently scored higher (worse) on all components of the DLQI. Dark skinned individuals with Vitiligo had maximum total impairment of $31.3 \%$ when compared to other group. Unmarried Patients showed greater impairment in symptoms and feeling and leisure component of DLQI when compared to married patients. Patients with chronicity greater than 5 yrs averaged $29.6 \%$ of maximum disability which is higher (worse) than those below 5 yrs $27.1 \%$ and also had the maximum score in treatment component $76.5 \%$. Slow responders (repigmentation less than $40 \%$ after one year of treatment) showed greater impairment in treatment section of DLQI $62.3 \%$ compared to rapid responders $26.19 \%$.

\section{Discussion}

Vitiligo can have a significant effect on the psychological well-being of the patient [11]. This is especially true for darker skinned patients as the contrast between the pigmented and depigmented skin can be quite drastic. In some cultures there is a stigma attached to having Vitiligo. Those affected with the condition are sometimes thought to be evil or diseased and are shunned by others in the community. People with Vitiligo may feel depressed because of their appearance has changed dramatically and the fact that they are not treated on par with normal individuals. In this study it was observed that Vitiligo affects all age groups of both sex. Interestingly, dark and light skinned individuals were found to be much less likely to suffer with Vitiligo compared to individuals with a brownish complexion. Most of the patients were in the second and third decade of their life. It is more acute in the case of young women and children; however, the majority of the Vitiligo cases are reported during stages of active development. About $50 \%$ of patients present before the age of 20 and nearly $70-80 \%$ present before 30 years of age. Although no age is immune to Vitiligo, the disease is very rarely observed at birth [12]. Vitiligo is reported more frequently in females than males, which may be the result of increased reporting rates in females due to greater social consequences in females affected by Vitiligo [13]. This is seriously felt among young unmarried women. Thus a young woman with vitiligo has little chance of getting married. A married women developing vitiligo after marriage shall have marital problems perhaps ending in divorce. Vitiligo lesions over face may be particularly embarrassing and the frustration of resistant lesions over exposed part of hands and feet can lead to anger and disillusionment.
In the present study scores on the DLQI ranged from 0-22 (mean \pm $\mathrm{SD}, 8.21 \pm 4.88)$, indicating poor quality of life in South Indian Vitiligo population when compared to the scores on the DLQI of Persian population that ranged from 0 to 24 (mean \pm SD,7.05 \pm 5.13 ) [9]. The mean DLQI score (8.21) in this study was higher than that reported in France (7.17), Belgium (6.9) and that of patients with Acne vulgaris (7.45), but lower than Psoriasis (10.53) or Atopic dermatitis (11.20) $[14,15]$. The mean DLQI score in this study was also higher than that obtained mean 7.3 and Kent and Al-Abadie's [16] study mean 4.82 but lower than Parsad et al. [17] (mean 10.6 this difference is due to the fact that south India has higher literacy rate and different culture as compared to north India). The association between poor QoL and depressive symptoms suggests that healthcare professionals should watch for possible depression in high-risk patients. Frequent complaint of embarrassment, despite low rates of poor self-esteem, suggests that while most patients cope well with their appearance, they continue to encounter unpleasant social interactions. Increasing public awareness about vitiligo to reduce unnecessary attention may benefit more than self-esteem management for vitiligo patients in South India [18].

Patients with non-segmental type of vitiligo reported more impairment than corresponding subgroups because of irregularly spread depigmented patches on the exposed part of the skin. Weiss et al compared the difficulties faced by patients with vitiligo with those with leprosy in India [19]. Salzer and Schallreuter reported that $75 \%$ patients found their disfigurement moderately or severely intolerable. These findings also support the relationship between stress and the development of vitiligo. Al-Abadie et al. [20] indicated that psychological stress increases levels of neuroendocrine hormones that affects the immune system and alters the level of neuropeptides, which may be the initial steps in pathogenesis of vitiligo. The chronic nature of disease, long term treatment, lack of uniformity, high cost and unsatisfactory pigmentation of therapy are usually very demoralizing for patients suffering from Vitiligo. Compliance for regular long term visits for PUVA/UVB therapy; side effects of immunosuppressive therapies are other limitation in treatment of Vitiligo patients, Interestingly in this study, an encouragingly huge section $(92.46 \%)$ of the patients responded to various degrees to the Unani treatment given to them at CRIUM and in most cases, the disease was found to limit itself for about 10 years after onset. Data collected from case records of these patients showed that the response to Unani treatment (with a mean $46 \%$ repigmentation) was remarkably better when compared to Ayurveda (12\%), Homeopathy (5.6\%) and Allopathy (5.8\%) treatment.

Our results show that in Vitiligo patient's quality of life is poor and it is strongly associated with their social well-being. Patients with high DLQI responded less favourably to given treatment indicating that psychological counselling and comfort could obtain a better treatment outcome. Papadopoulos et al. [21] have shown that counselling can help to improve body image, self-esteem and quality of life of patients with Vitiligo, also having positive effect on the course of the disease. A recent study which measured the frequency and number of stressful life events occurring over a specified period among Vitiligo and alopecia areata suggested that such patients endured a significantly higher number of stressful life events than do controls suggesting that psychological distress may have contributed to the onset of Vitiligo and alopecia areata [22]. Hence improving quality of life in this group should be taken as an important task by the dermatologist while treating Vitiligo patients.

\section{Acknowledgement}

We are grateful to staff and subjects for help in compiling the data and senior dermatologist at CRIUM Hyderabad, Dr. M.A. Waheed for his kind cooperation. 


\section{References}

1. Lerner AB (1959) Vitiligo. J Invest Dermatol 32: 285-310.

2. Krüger C, Schallreuter KU (2012) A review of the worldwide prevalence of vitiligo in children/adolescents and adults. Int J Dermatol 51: 1206-1212.

3. Behl PN, Aggarwal A, Srivastava G, Behl PN, Srivastava G, et al. Practice of Dermatology. (9thedn). CBS Publishers, New Delhi, India.

4. Valia AK, Dutta PK (2001) IADVL Text book and Atlas of Dermatology. Bhalani Publishing House, Mumbai, India.

5. Taïeb A (2000) Intrinsic and extrinsic pathomechanisms in vitiligo. Pigment Cell Res 13: 41-47.

6. Njoo MD, Westerhof W (2001) Vitiligo. Pathogenesis and treatment. Am J Clin Dermatol 2: 167-181.

7. Singh S, Singh U, Pandey SS (2012) Serum concentration of IL-6, IL-2, TNF- $\alpha$, and IFNY in Vitiligo patients. Indian J Dermatol 57: 12-14.

8. Kumar S, Singh A, Prasad RR (2011) Role of histopathology in vitiligo. J Indian Med Assoc 109: 657-658.

9. Taneja A (2002) Treatment of vitiligo. J Dermatolog Treat 13: 19-25.

10. Finlay AY, Khan GK (1994) Dermatology Life Quality Index (DLQI)--a simple practical measure for routine clinical use. Clin Exp Dermatol 19: 210-216.

11. Mechri A, Amri M, Douarika AA, Ali Hichem BH, Zouari B, et al. (2006) Psychiatric morbidity and quality of life in Vitiligo: a case controlled study. Tunis Med 84: 632-635.

12. Gauthier Y, Cario-Andre M, Lepreux S, Pain C, Taïeb A (2003) Melanocyte detachment after skin friction in non lesional skin of patients with generalized vitiligo. Br J Dermatol 148: 95-101.
13. Cho S, Kang HC, Hahm JH (2000) Characteristics of vitiligo in Korean children. Pediatr Dermatol 17: 189-193.

14. Basra MK, Fenech R, Gatt RM, Salek MS, Finlay AY (2008) The Dermatology Life Quality Index 1994-2007: a comprehensive review of validation data and clinical results. $\mathrm{Br} J$ Dermatol 159: 997-1035.

15. Kostopoulou P, Jouary T, Quintard B, Ezzedine K, Marques S, et al. (2009) Objective vs. subjective factors in the psychological impact of vitiligo: the experience from a French referral centre. Br J Dermatol 161: 128-133.

16. Kent G, al-Abadie M (1996) Factors affecting responses on Dermatology Life Quality Index items among vitiligo sufferers. Clin Exp Dermatol 21: 330-333.

17. Parsad D, Pandhi R, Dogra S, Kanwar AJ, Kumar B (2003) Dermatology Life Quality Index score in vitiligo and its impact on the treatment outcome. $\mathrm{Br} J$ Dermatol 148: 373-374.

18. Salzer BA, Schallreuter KU (1995) Investigation of the personality structure in patients with vitiligo and a possible association with impaired catecholamine metabolism. Dermatology 190: 109-115.

19. Weiss MG, Doongaji DR, Siddhartha S, Wypij D, Pathare S, et al. (1992) The Explanatory Model Interview Catalogue (EMIC). Contribution to cross-cultural research methods from a study of leprosy and mental health. $\mathrm{Br} \mathrm{J}$ Psychiatry 160: 819-830.

20. Al'Abadie MS, Kent GG, Gawkrodger DJ (1994) The relationship between stress and the onset and exacerbation of psoriasis and other skin conditions. Br J Dermatol 130: 199-203.

21. Papadopoulos L, Bor R, Legg C (1999) Coping with the disfiguring effects of vitiligo: a preliminary investigation into the effects of cognitive-behavioura therapy. Br J Med Psychol 72: 385-396.

22. Gupta MA Gupta AK, Watteel GN (1997) Stress and alopecia areata: psychodermatologic study. Acta Derm Venereol 77: 296-298. 